\title{
Single-Channel Region-Based Speller for Controlling Home Appliances
}

\author{
Praveen Kumar Shukla, National Institute of Technology, Raipur, India \\ Rahul Kumar Chaurasiya, Malaviya National Institute of Technology, Jaipur, India \\ Shrish Verma, National Institute of Technology, Raipur, India
}

\begin{abstract}
The brain-computer interface (BCI) system uses electroencephalography (EEG) signals for correspondence between the human and the outside world. This BCI communication system does not require any muscle action; hence, it can be controlled with the help of brain activities only. Therefore, this kind of system is helpful for patients, who are completely paralyzed or suffering from diseases like ALS (Amyotrophic Lateral Sclerosis), and spinal cord injury, etc., but having a normal functioning brain. A region-based P300 speller system for controlling home electronic appliances is proposed in this article. With the help of the proposed system, users can control and use appliances like an electronic door, fan, light, system, etc., without carrying out any physical movement. The experiments are conducted for five, ten, and fifteen trails for each subject. Among all classifiers, the ANN classifier provides the best off-line experiment accuracy of the order of $80 \%$ for fifteen flashes. Moreover, for the control translation, the Arduino module is also designed which is low cost and low power-based and physically controlled a device.
\end{abstract}

\section{KEYWORDS}

ANN, Brain-Computer Interface (BCI), EEG Signal, Home Appliance, Region-Based Speller, Single Channel

\section{INTRODUCTION}

People suffering from neuromuscular diseases like brain stroke and Amyotrophic Lateral Sclerosis (ALS) have very limited muscle-based control capabilities; hence they are heavily dependent on secondary caregivers. For such patients, Brain-computer interface (BCI) is a useful approach for interaction with the outside world. This technology provides communication between the human brain and the external environment. BCI systems acknowledge the subject's intentions and convert these brain patterns and signals into control command (Mak \& Wolpaw, 2009). Once the signals are converted into control commands they are used in a wide range of applications such as controlling a wheelchair, 2-D cursor, keyboard, mobile robot, home appliances (Hoffmann, Vesin, Ebrahimi, \& Diserens, 2008), etc. BCI-based home appliances control system is an important application that will give an easy life to a patient who has a neuromuscular disease. Moreover, BCI also reduces the cost of care and dependency on others. The use of Brain waves for controlling the support system 
has been an active research and development area for about the last 10 years. Some of the major contributions in $\mathrm{BCI}$ are now described.

Brain patterns are analyzed by acquiring electroencephalography (EEG) signals. Both invasive and non-invasive techniques are used to acquire EEG signals (Ramadan \& Vasilakos, 2017). Non-invasive methods are more popular for BCI as do not require surgical implantation of the devices. The ease of access along with the painless acquisition of EEG signals inspired the author to use the noninvasive method. There are six types of signals generated from the scalp which is dependent on the frequency band. A P300 potential is one of the most important components of EEG which works on the principle of odd-ball paradigms (Farwell \& Donchin, 1988). It is used to transfer subject intention into input command. For EEG-based BCI, several other signal patterns are used. These include steady-state visually evoked potentials (SSVEPs) (Zhang, Yu, Jiang, Wang, \& Qin, 2019), motion-onset visually evoked potentials (MEPs) (T. Ma et al., 2017), motor imagery (MI)(Jais, Mansor, Lee, \& Fauzi, 2017), hybrid signal (Masud, Baig, Akram, \& Kim, 2017), etc. BCI-analysis is a useful approach for clinical applications like understanding the need \& requirement of totally disabled patients in hospitals. Many paradigms like single character (SC)(Pan, Li, Gu, \& Yu, 2013), row/column (RC)-based (Farwell \& Donchin, 1988), region-based (RB)(Fazel-Rezai \& Abhari, 2009), checkboard (CB) (Townsend et al., 2010), rapid serial visual presentation (RSVP)-based (Acqualagna \& Blankertz, 2013) have been investigated using BCI analysis.

Controlling home appliances using EEG-signal based approach in a real environment is a complex task due to various difficulties like the cost of software and hardware, stability in system performance, convenience for users, and technical challenges that occurred in real life. Some of the real-life examples for BCI-based home appliances control systems are wireless BCI-based system especially P300 and SSVEP based signal translation, Bluetooth-based wireless transmission of EEG signal, infra-red (IR) technology, TCP/IP communication and UPnP (Universal plug and play). But limitation these systems are that they depend upon the availability of the internet and many times they are not capable to interact with many devices at a time. Several research publications discuss about single appliance control, for example, fan device (Wang, Lv, Wen, He, \& Wang, 2016), TV (Hsieh, Sun, Yeh, \& Pan, 2017a), cooling system (Hsieh, Sun, Yeh, \& Pan, 2017b), door (Alrajhi, Alaloola, \& Albarqawi, 2017), buzzer and music system (Chowdhury, Kashem, Hossan, \& Hasan, 2017; Tseng, Wang, Lin, \& Hsieh, 2012). Some researchers proposed multiple appliance control (TV, Fan, AC, Door, light, etc..), virtual reality-based (Holzner, Guger, Edlinger, Gronegress, \& Slater, 2009), microcontroller-based (Alshbatat, Vial, Premaratne, \& Tran, 2014), FPGA-based (Belwafi, Ghaffari, Djemal, \& Romain, 2017), infrared-based (IR) design (Corralejo, Nicolás-Alonso, Álvarez, \& Hornero, 2014), Bluetooth-based (Lin, Lin, Lin, \& Chang, 2014), web server-based (Aydın, Bay, \& Güler, 2016) have been implemented.

Some attempts have been made to control home appliances through eye blink signals. (Wahy \& Mansor, 2010a) proposed a design to control light bulbs using eye blinks. (Uma \& Sheela, 2017) used a graphical user interface-based system to control the left and right direction of wheelchair and home appliances. (Usakli \& Gurkan, 2010) suggested a design to operate the keyboard control system through eye blink detection. (J. Ma, Zhang, Cichocki, \& Matsuno, 2014) proposed prototype for robot control using different eye movements and ERP-based. (He \& Li, 2017) suggested a single channel-based EOG speller using eye blink for character recognition. (Khushaba, Kodagoda, Lal, \& Dissanayake, 2011) have been proposed to extract numerous cognitive signals like eye blink which are used to detect drowsiness from the driver. The reduction of eye blink kind artifacts from EEG signals is an important preprocessing step in designing of EEG-based home appliances control system to enhance the efficiency of these systems. There are various methods are used for detection and reduction of eye blink artifacts from EEG signals which are following: Detection of eye blink artifacts, using a discrete wavelet transform (DWT) (Tibdewal, Fate, Mahadevappa, \& Ray, 2015), suppression of eye blink artifacts by cross-correlation and empirical mode decomposition (EMD) (Patel, Janawadkar, Sengottuvel, Gireesan, \& Radhakrishnan, 2016), regression (Hillyard \& Galambos, 1970), blind 
source separation (BSS) (Sweeney, Ward, \& McLoone, 2012), principal component analysis (PCA) (Casarotto, Bianchi, Cerutti, \& Chiarenza, 2004), wiener filtering (Somers, Francart, \& Bertrand, 2018), sparse decomposition (Donoho, 2001), hybrid method- wavelet and ICA (Pesin, 2007), EMD and BSS (Soomro, Badruddin, Yusoff, \& Jatoi, 2013).

\section{Motivation and Requirements}

In India, 2.21 percent of the total population is disabled (Velayutham, Kangusamy, Joshua, \& Mehendale, 2016). According to Census 2011, in India, 20\% are having movement disability, 19\% are with the disability in seeing, and $8 \%$ have multiple disabilities. The brain-computer interface communicates with those people, whose motor cell is damaged and who are unable to perform any muscle activity. Therefore, by using BCI, they can interact with the external environment and access their daily needs like a wheelchair, home appliance, 2D-3D cursor control, etc. BCI-based home appliances system can solve the above-mentioned problems of disable and elderly people. Following are the design requirements and objectives of such BCI based system:

- To design a signal acquisition system for BCI-based home appliances and control system.

- To select accurate classifier and design a suitable and quick response hardware module.

- To make a cost-effective system that can control the maximum number of devices.

\section{Proposed Work Based on the State of Art}

In this study, a BCI-based control system for home appliances is proposed. In the proposed system classified signal is converted into a command signal. The control interface includes two different stages namely 1) BCI interface and 2) Control translation. BCI interface normally has four stages namely, data collection, preprocessing, feature extraction, and classification. In this proposed work, a novel data acquisition system is designed, dedicatedly for paralyzed people. In this state-of-art proposed work, an eye blink based home appliance control system is designed. The proposed system uses Arduino-UNO based hardware module to make the proposed system cost-effective. The system can handle a maximum number of appliances that include compatibility with electrical devices. Moreover, in this paper, a region-based speller system is proposed, which can control appliances like fan, light bulb, electric heater, etc. using eye blink signals. The region-based speller contains 2-panel menus on which the required option can be selected through an eye blink signal. In the subpanel menu, there is a back option that is selected through eye blink; with this option, the user can come back to the main menu. The proposed system will help to reduce the dependency of the paralyzed patient on caregivers at low cost and enhance their lifestyle. Furthermore, we have also implemented an eye blink artifact removal method to enhance the performance of the proposed system. The proposed block diagram of such a BCI-based system to control the home appliances as proposed is shown in Figure 1.

The rest of the article is structured as follows: The next section explains about material and methods and classifiers. Then the data acquisition section explains, preprocessing steps, feature extraction methods, and proposed system architecture. Afterward, the result section represents the evaluation of the proposed method. The discussion and future scope section are dedicated to the discussion and finally concludes the study.

\section{MATERIAL AND METHOD}

The working principle of the proposed BCI-based home appliances control system has five subtasks viz data collection, preprocessing of the acquired EEG signals, classification of blink and non-blink signals, correct target appliance detection and finally appliances connected to the hardware instrument. The working principle of the proposed approach is shown in Figure 2. 


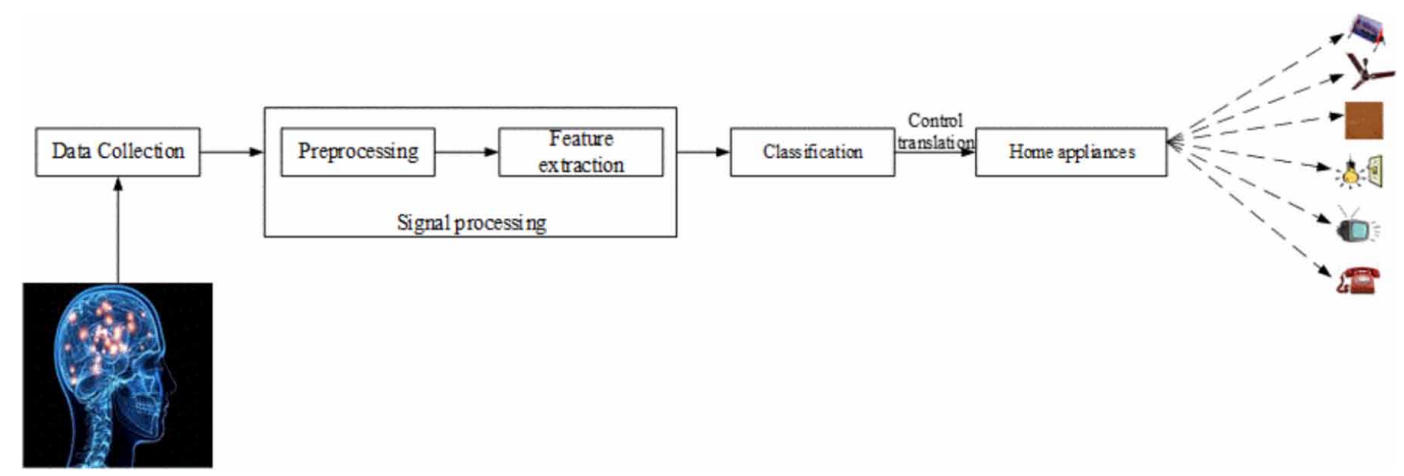

Figure 2. Working principle of $\mathrm{BCl}$-based control of home appliances system

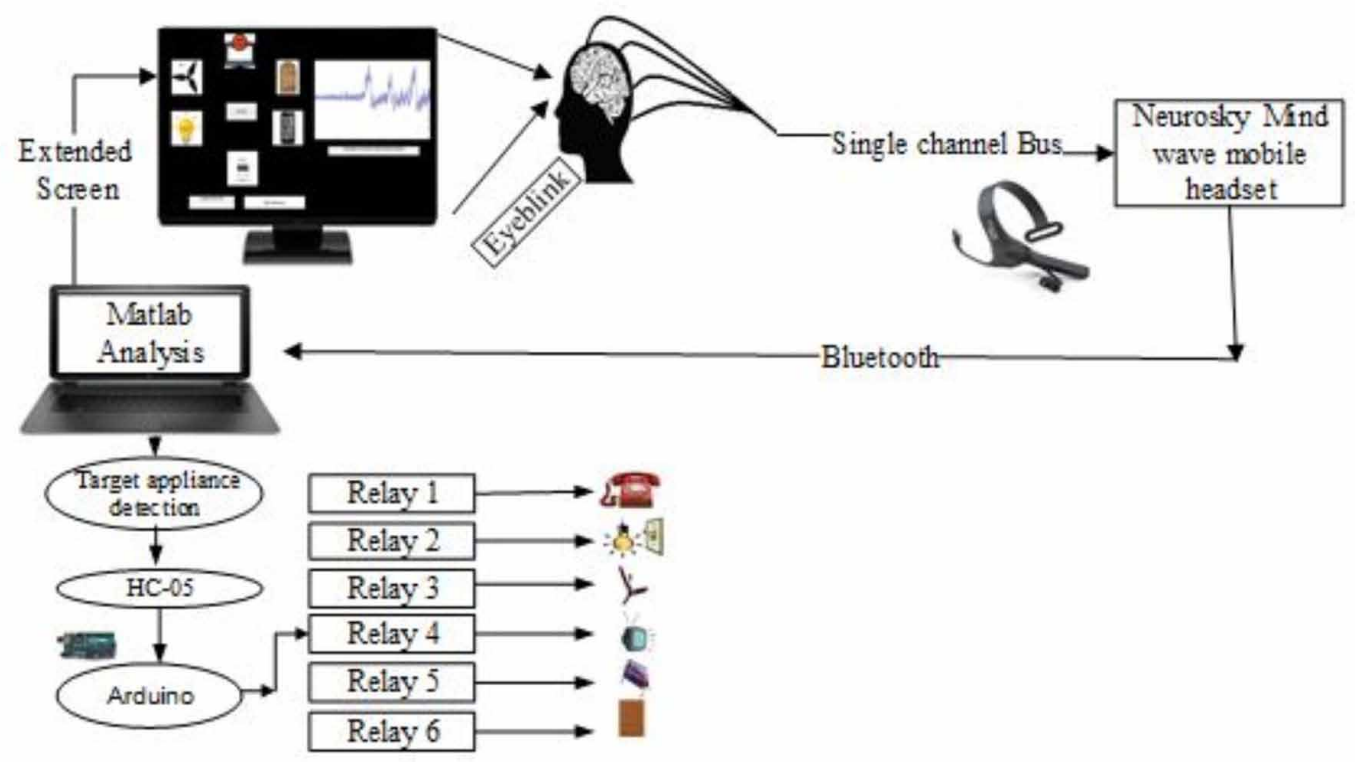

\section{Proposed Region-Based Paradigms for Controlling of Home Appliances System}

Generally in region-based paradigms (RBP), there is the main screen that is connected with user and again the screen subdivides into many sub-regions where the item to facilitate the user. For the selection of the target icon from the main panel, RBP operates on two levels, the former is called the main panel and the scatter is called a sub-panel. These panels are used for the selection of stimuli presentation and target selection. The main panel consists of many options for the selection of various home appliances like TV, door, mobile, light bulb, fan, electric heater, etc. Figure 3 shows the proposed GUI for the region-based speller system. The upper screen shows the main menu and down one display sub-regions. Once a selection is made from any of the 6 options through eye blink, the display is redirected to the selected sub-panel menu. Depending on the detected appliances, the main paradigm will be redirected to one of the 6 possible paradigms to control mobile-phone/TV/ door/light-bulb/electric-heater/fan. 
Figure 3. Proposed region-based (RB) paradigms for the home environment

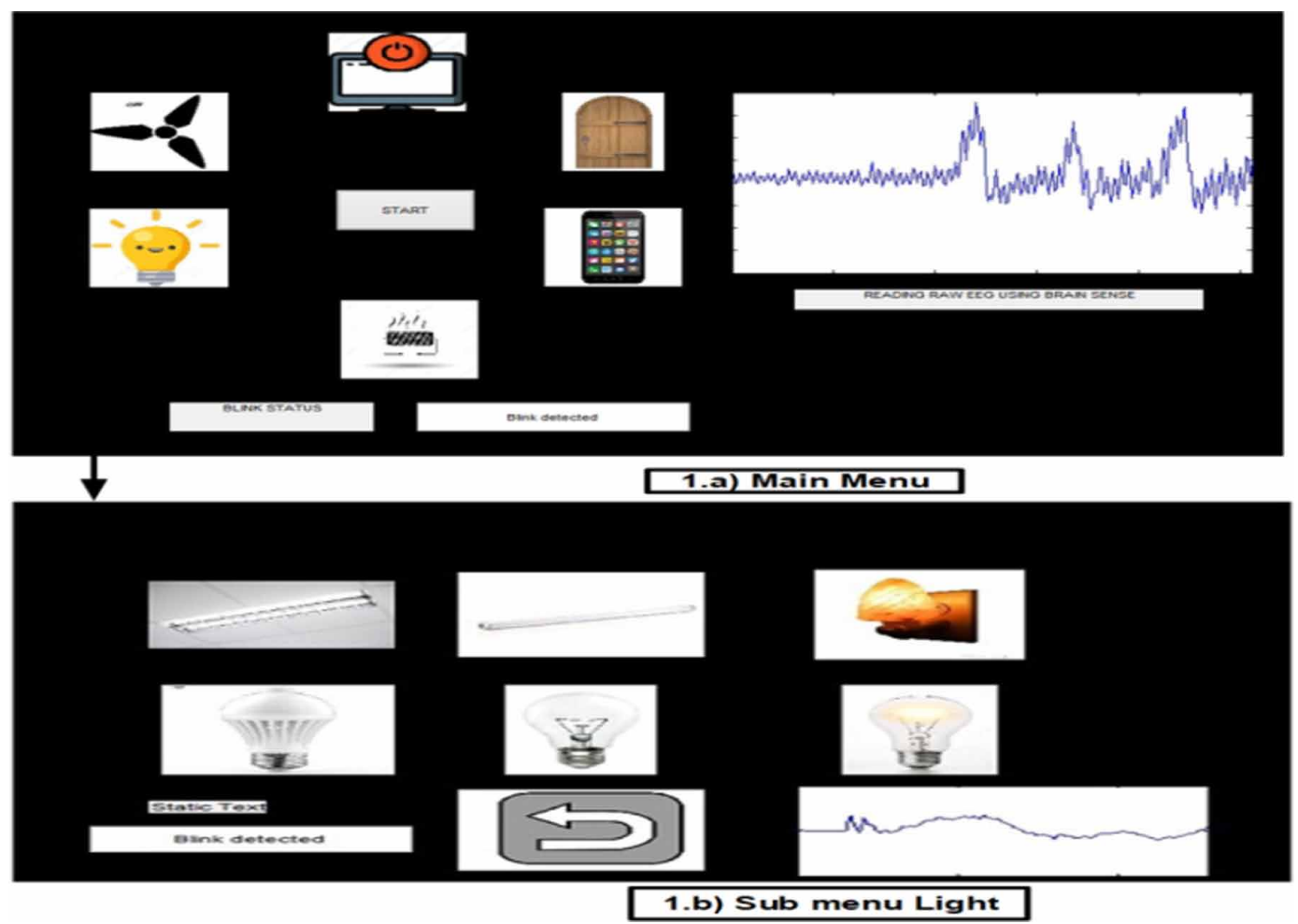

Figure 4(a) shows the paradigm to control mobile that contains forward, backward, the main menu, call orientation, call termination and hash button. Figure 4(b) shows the paradigm to control door that contains door close, door open, key inserted and key not inserted, door locked, door not locked. Figure 4(c) shows the paradigm to control a fan that contains fan on, fan off, fan running on speed 0-3. Figure 4(d) shows the paradigms to control the light that contains Tub light on/off, night bulb on/off and light bulb on/off. Figure 4(e) shows the paradigms to control heater that contains on/off, heater ignition started, and heater full ignite, temperature low/high. Figure 4(f) shows the paradigms to control TV that contains TV on/off, volume up, volume down channel up, channel down, and mute options.

Classification of acquired signals helps in predicting blink signals in the target region. Blink and non-eye blink signal prediction is a binary classification task. Considering the challenge of classification in the region based (RB), we are applying artificial neural network (ANN) classifier, support vector machine (SVM), and Linear discriminate analysis (LDA) classifiers $\mathrm{KNN}$ and Bayes classifier.

\section{Artificial Neural Network (ANN)}

Figure 5 shows a commonly used model called the multi-layered perceptron (MLP). it comprises one layer of input and one layer of output and a hidden layer between input and output (Mitra \& Pal, 1995). Each layer uses multiple neurons and each neuron in a layer is linked with distinct weights to the neurons in the neighboring layer. Except for the input layer, each neuron gets signals from the prior layer's cells linearly weighted with neuron-to-neuron interconnect values.

It is presumed that a total of $\mathrm{N}$ sets of training data will be accessible. Inputs $\left\{\mathrm{X}_{1,} \mathrm{X}_{2}, \mathrm{X}_{3} \ldots \ldots \ldots, \mathrm{X}_{N}\right\}$ are fed to the input layer. The ANN is trained to respond to the 
Figure 4a. Sub-regions

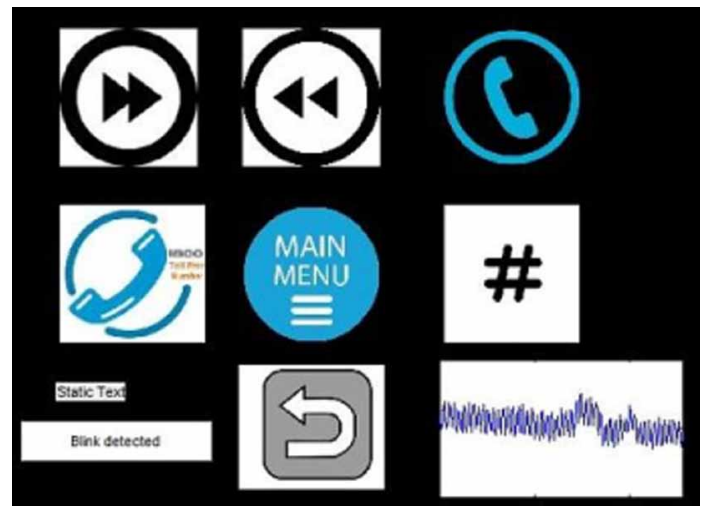

Figure 4b. Door control

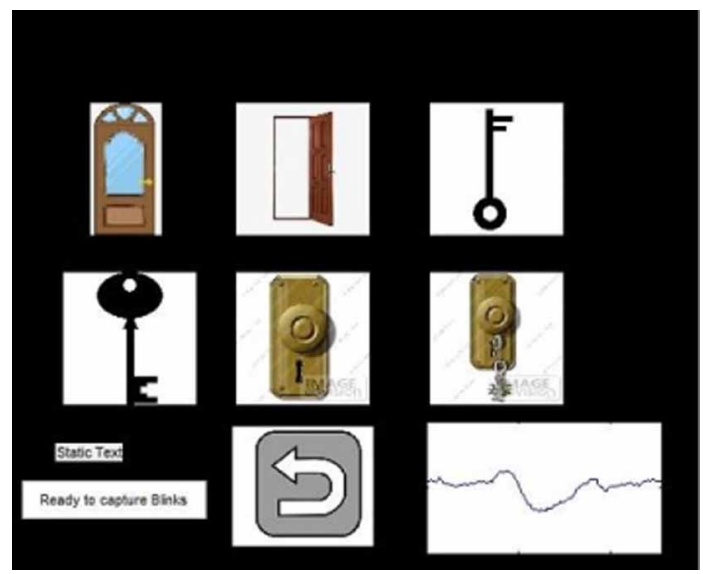

Figure 4c. Fan control

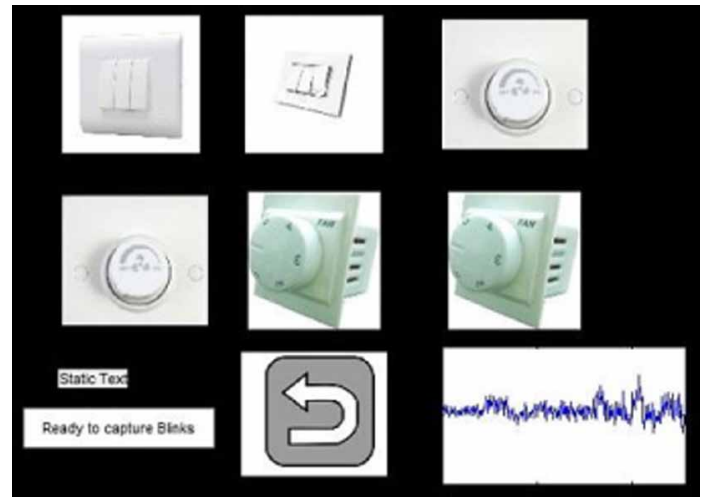


Figure 4d. Light control

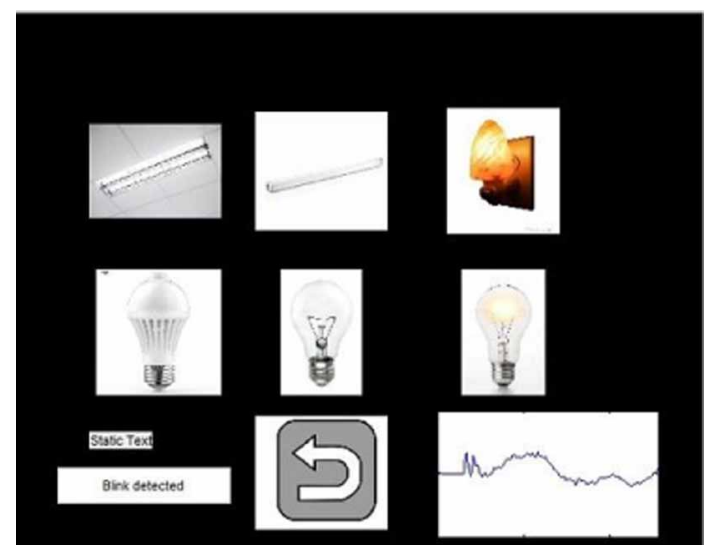

Figure 4e. Heater control

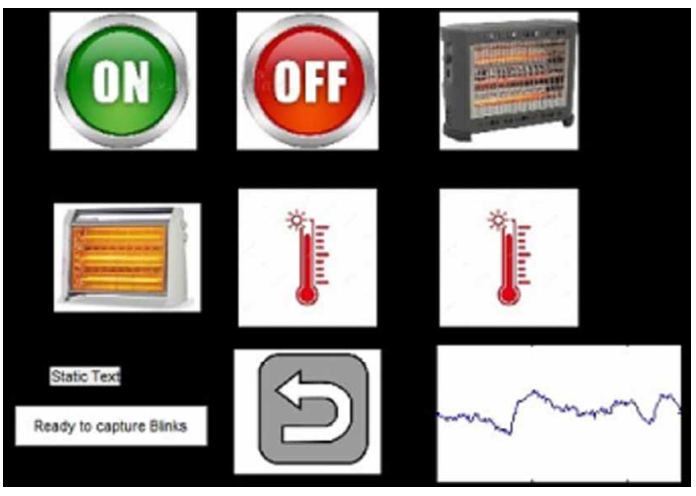

Figure 4f. TV control

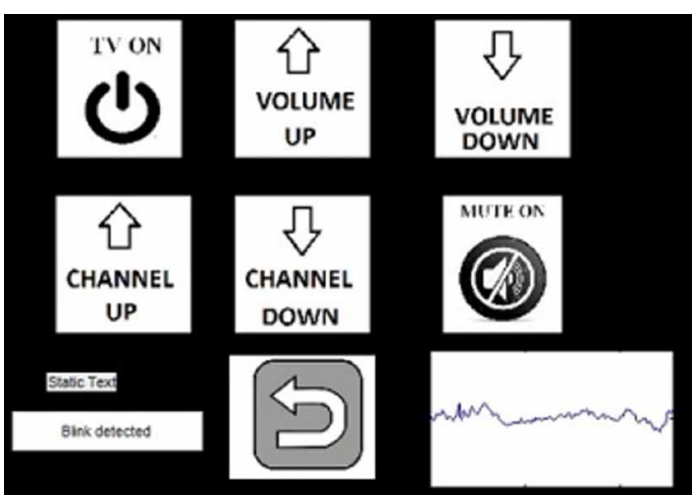




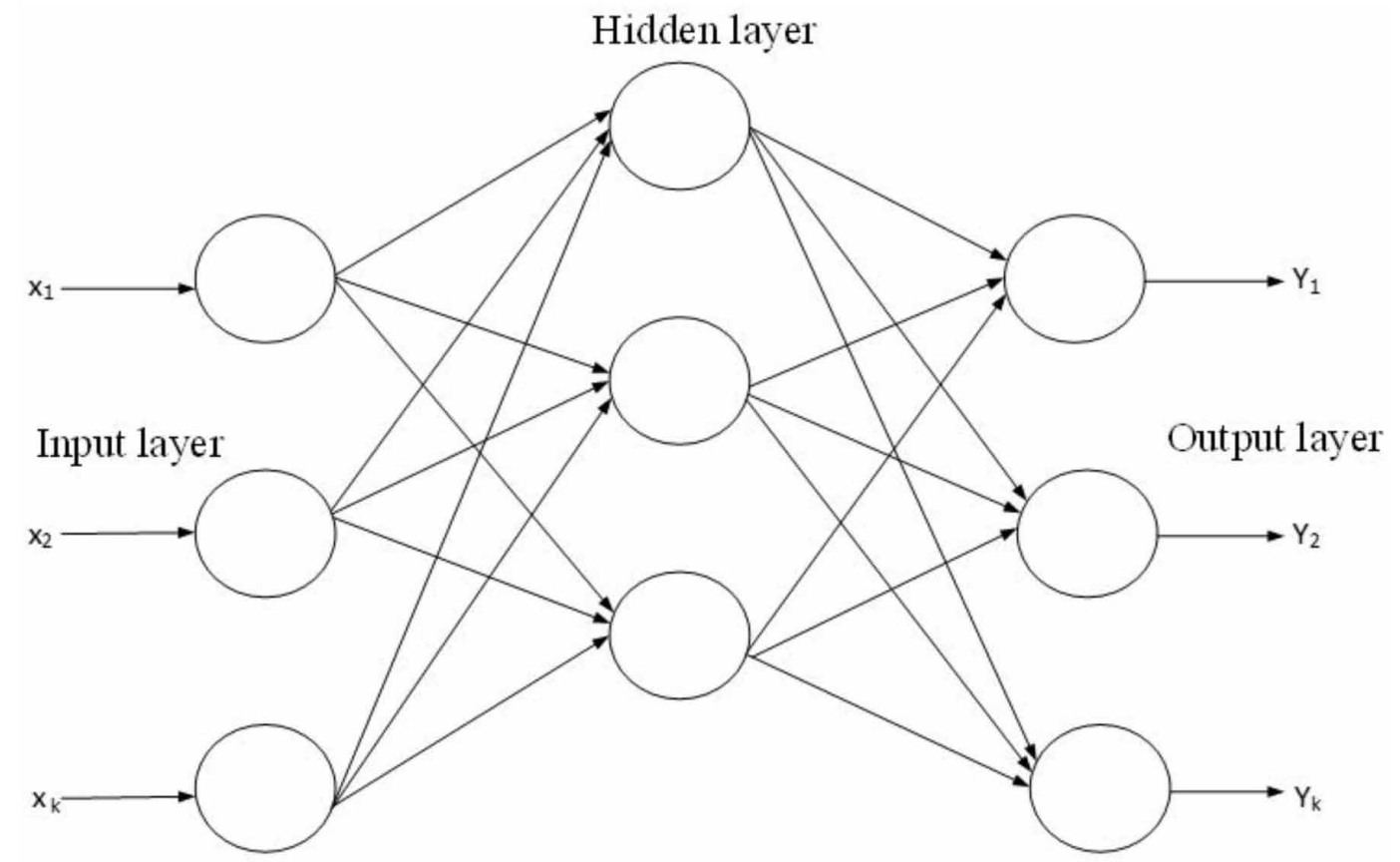

corresponding target vectors $\left\{Y_{1} \mathrm{Y}_{2}, \mathrm{Y}_{3} \ldots \ldots \ldots, \mathrm{Y}_{N}\right\}$ on the output layer. The training goes on until a certain stop-criterion is met. Training is typically stopped when the average error is less than a predetermined threshold between the desired and actual outputs of the neural network over the $\mathrm{N}$ training data sets. The weights of the network $\{\mathrm{Wij}\}$ are adjusted to decrease the output error according to the difference between the generated and the actual outputs. A node's activation function determines the neuron's output given input or an array of inputs.

The output of neuron $\mathrm{k}, \mathrm{Y}_{\mathrm{k}}$ connected from the input of neuron layer $\mathrm{j}$ is:

$$
Y_{k}=f\left(\sum_{j} w_{k j} \cdot O_{j}\right)
$$

where $\mathrm{w}_{\mathrm{kj}}$ is interconnection weight, $\mathrm{O}_{\mathrm{j}}$ is the output of a neuron in previous layers and $\mathrm{f}$ is sigmoid function $f(a)=\frac{1}{1+e^{-a}}$.

The loss function can be defined as:

$$
E\left(w_{k j}\right)=\frac{1}{2}\left(y_{\text {true }}-y_{k}\right)^{2}
$$

where $\mathrm{y}_{\text {true }}$ is the correct output for the input $\mathrm{x}_{\mathrm{i}}$.

Weights of the final layer are updated by the gradient descent algorithms (Qian, 1999): 


$$
\frac{\partial E}{\partial w_{k j}}=\frac{\partial E}{\partial y_{k}} \cdot \frac{\partial y_{k}}{\partial a_{k}} \cdot \frac{\partial a_{k}}{\partial w_{k j}}=-\left(y_{\text {true }}-y_{k}\right) y_{k}\left(1-y_{k}\right) O_{j}=-\delta_{k} O_{j}
$$

where $\delta_{k}=\left(y_{\text {true }}-y_{k}\right) y_{k}\left(1-y_{k}\right)$ and $\mathrm{a}_{\mathrm{k}}$ is input for sigmoid activation function (Han \& Moraga, 1995).

So weight change is:

$$
\Delta W_{k j}=\eta \cdot \delta_{k} \cdot O_{j}
$$

where $\eta$ is the adaption gain.

Error in the hidden layer is given by:

$$
\delta_{j}=O_{j}\left(1-O_{j}\right) \sum_{k} w_{k j} \cdot \delta_{k}
$$

and again weight change is given by:

$$
\Delta W_{j i}=\eta \cdot \delta_{j} \cdot x_{i}
$$

where $\mathrm{x}_{\mathrm{i}}$ is input to the hidden layer.

\section{Support Vector Machine (SVM)}

SVM classifier provides good generalization capability. If the data is linearly separable, then SVM classifiers are in two hyperplanes such that separation margin's is maximized between two classes of the used dataset. The function involves optimizing the separation margin and managing error point, which is allowed in the process called stack variables, for the non-linearly separable dataset. This issue is considered an optimization problem and is resolved by Lagrange's multipliers. As a result, for novel data points $\mathrm{x}$ consider a linear, discrete function as shown in equation (7).

$\mathrm{F}(\mathrm{x})=\sum_{i=0}^{n} y_{i} \lambda_{i}\left(x . x_{i}\right)+\mathrm{b}$

where $x_{i}, \mathrm{i}=1,2 \ldots \ldots \mathrm{N}$ are trainning data points with $y_{i} \in\{-1,1\}, i=1,2 \ldots N, \lambda_{i} i=1,2 \ldots . \mathrm{N}$.

It is observed that by replacing dot product $\left(x . x_{i}\right)$ with a Kernal function $\mathrm{K}\left(\boldsymbol{x} . \boldsymbol{x}_{i}\right)$ this equation (7) converted into equation (8) which is called radial basis function (RBF) (Dhanalakshmi, Palanivel, \& Ramalingam, 2009):

$\mathrm{K}\left(\mathrm{x} \cdot \mathrm{x}_{\mathrm{i}}\right)=\exp \left(-\frac{\left\|\mathrm{x}-\mathrm{x}_{\mathrm{i}}\right\|^{2}}{2 \tilde{\mathrm{A}}^{2}}\right)$ 


\section{Linear Discriminant Analysis (LDA)}

LDA's concept is to discover a hyperplane maximizing the distance between means of two classes and minimizing the variance of the interclass. Classical LDA aims at optimizing the conversion by minimizing the distance between classes and maximizing the distance between classes at the same time, thus reaching maximum discrimination. By calculating the Eigen decomposition on the scatter matrices, the ideal transformation can be easily discovered.

\section{DATA ACQUISITION}

All participants involved in this study suffer from motor impairments disease. The details of all nine participants are shown in Table 1. Data were acquired through a single-channel Neurosky headset as shown in Figure 6. In this screenshot, the subject wears a single channel neurosky mind wave mobile headset. This device permits associations with apps like BCI2000, Mat lab and digitized forehead brainwave signals. This device is able to measure multiple states of the mind simultaneously. The data were recorded with nine subjects ages range 21 to 40 at the research lab of the national institute of technology, Raipur India. The data samples collected for a time period of (0 to 600) ms, with a sampling rate of $512 \mathrm{~Hz}$. MATLAB software was used for designing of region-based paradigm.

Figure 6. Screenshot of the participant wearing Neurosky headset

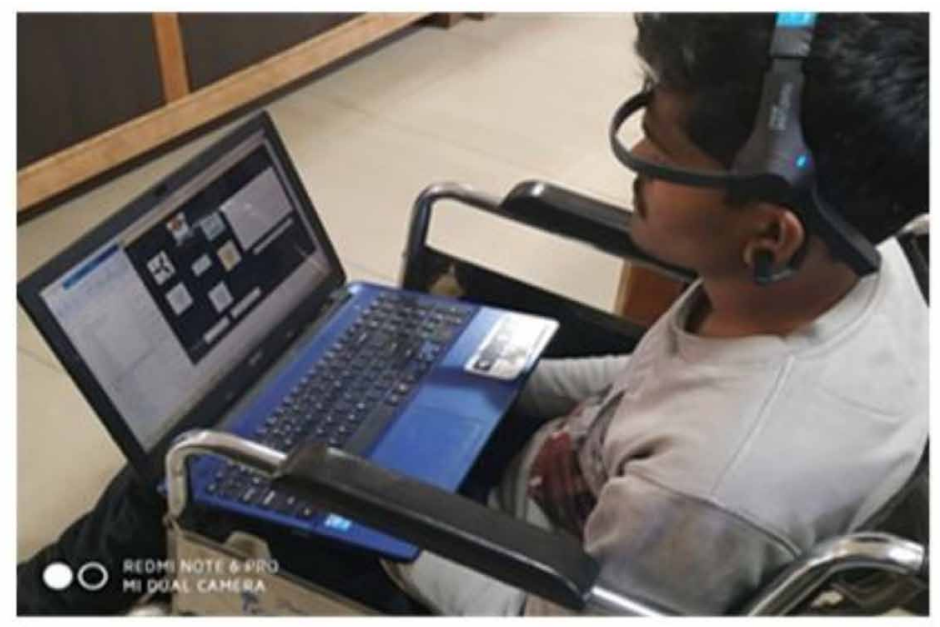

In the main panel, six appliances (icon) are presented which are shown in Figure 3. Icons were shown in random order to the subjects for a fixed amount of time. The subject was asked to do a single eye blink for activating the device. Once the subject selects a device according to his needs, its sub-panel will be open. In subpanel there are 6 icons for selection, users can select according to their needs by a single eye blink. In the subpanel, the icon was also shown in random order. Subpanel practiced the same method as the main panel. After that subject can select the 'go back' option for going back to the main menu by using a single eye blink. Here, the appliance status in the main panel menu was the same as previously. The subject can change device status through selection, using a single eye blink. The experiments were performed in a silent room. In training sessions, the subject was requested to complete 18 tasks ( 36 selections) for both trainings as well as testing sessions. In the testing session, classes were not defined. The multi-trail analysis was carried out in this study. 
Table 1. Summary of all participants

\begin{tabular}{|l|l|l|l|l|l|l|}
\hline \multicolumn{1}{|r|}{ Sub.no } & \multicolumn{1}{|c|}{ Patient } & \multicolumn{1}{|c|}{ Age } & \multicolumn{1}{|c|}{ Sex } & \multicolumn{1}{c|}{ Diagnosis } & $\begin{array}{c}\text { Time since } \\
\text { Injuries } \\
\text { (Months) }\end{array}$ & $\begin{array}{c}\text { Motor } \\
\text { impairment } \\
\text { degree }\end{array}$ \\
\hline 1. & Subject 1 & 30 & M & SCI & 11 & A \\
\hline 2. & Subject 2 & 35 & M & SCP & 5 & M \\
\hline 3. & Subject 3 & 40 & F & SCI & 16 & S \\
\hline 4. & Subject 4 & 25 & M & LD & 20 & M \\
\hline 5. & Subject 5 & 25 & M & LD & 25 & M \\
\hline 6. & Subject 6 & 30 & M & SCI & 5 & S \\
\hline 7. & Subject 7 & 35 & M & SCP & 10 & S \\
\hline 8. & Subject 8 & 33 & M & LD & 15 & M \\
\hline 9. & Subject 9 & 32 & M & SCI & 20 & M \\
\hline
\end{tabular}

SCl: Spinal cord injury, SCP: Spastic cerebral palsy, LD: Locomotive diseases

Motor impairment degree: absent $(A)$, minor $(m)$, modest $(M)$, severe $(S)$, profound $(P)$

We collected data into 5, 10 and 15 trails. The time required for completion of 15 trails is 27 seconds $\left(15^{*} 6^{*} 300 \mathrm{~ms}\right)$. To switch between levels the speller needs 2 seconds of time. Total time needed in 56 seconds for completing the task.

\section{Artifacts and Eye Blink Detection from EEG Signals}

EEG plays a significant role in recognizing brain activity and behavior. However, the electrical exercise recorded is continually contaminated with artifacts and affects the EEG signal analysis. There are various types of artifacts that come from the body itself (eye blink, muscles, and heartbeat) which are categories as intrinsic artifacts. There is an external artifact that comes from experimental error (electrode misplacement, cable misplacement) which is classified as extrinsic artifacts. In some instances, eye blinking artifacts are noises because EEG information collected with open eyes can be delivered with such artifacts. In this research, however, the eye blinking is the necessary parameter to activate the home appliances control system.

During the experiment, the data were saved by single-channel (Fp1) while the user intentionally caused an eye blink. Since we are collecting frontal region data, this portion of data is best suitable for blink detection. For the detection of eye blink signals, we have applied the thresholding method. Typically, eye blinks are defined by peaks with comparatively high voltages. They are often situated by setting a limit for all activities which exceed the threshold value and classifying it as eye blinks. There is also some variation in the amplitude of a particular individual's peaks, more variability among distinct subjects. Eye blinks can be categorized as small blinks if the blink period is less than $300 \mathrm{~ms}$ with a limit value of $\pm 4 \mu \mathrm{V}$. The detection and removal of small blink are necessary to maintain the peak amplitude of EEG signals. In the proposed RB home appliances control system, once eye blink signals (for example: single, double and triple) detected to the corresponding region particular appliances will activate.

\section{Preprocessing, Feature Extraction and Classification}

\section{Filtering}

EEG signals are extremely weak and are affected by various types of noise and impairments that must be carefully removed. Differential amplifiers are used to reduce the effect of common noise on the electrodes. Each electrode is connected to the input of a differential amplifier. A standard 
device reference electrode connects the other input of each differential amplifier. Such amplifiers, with extremely high peaks, are very low-noise amplifiers. There are various common filters, for example, low pass, high pass, band-stop, bandpass, notch, comb and all-pass filter which depends upon frequency responses. There are some linear filters, for example, Chebyshev filter, Butterworth filter, Elliptic filter, and Bessel filter are commonly used filters for EEG noise removal. The collected (EEG signal) was transferred to the PC by using Bluetooth at a baud rate of 57600. A built-in notch filter in the neurosky mind wave headset removes the noise in the $50-60 \mathrm{~Hz}$ band. But we need filtering to remove known noise frequency such as heartbeat and noise from muscles. Subsequently, an 8-order Chebyshev bandpass filter was used for filtering purposes of each extracted signal with a cutoff frequency range between 1 to $10 \mathrm{~Hz}$ and the data was decimated.

\section{Segmentation}

The data (EEG signal) collected from a single-channel neurosky mind wave mobile headset was segmented into one second time window for each stimulus. A feature vector was extracted for determining the target region using eye blink. After segmentation, six feature vectors namely X1 to X6 were created (shown in Figure 7). After the extraction of feature vectors, classification of eye blink (class labal+1) and non-eye blink (class label-1) was performed. In binary class classification problem one class belongs to blink signals and the other five classes belong to non-blink signals.

Figure 7. Segmentation of features
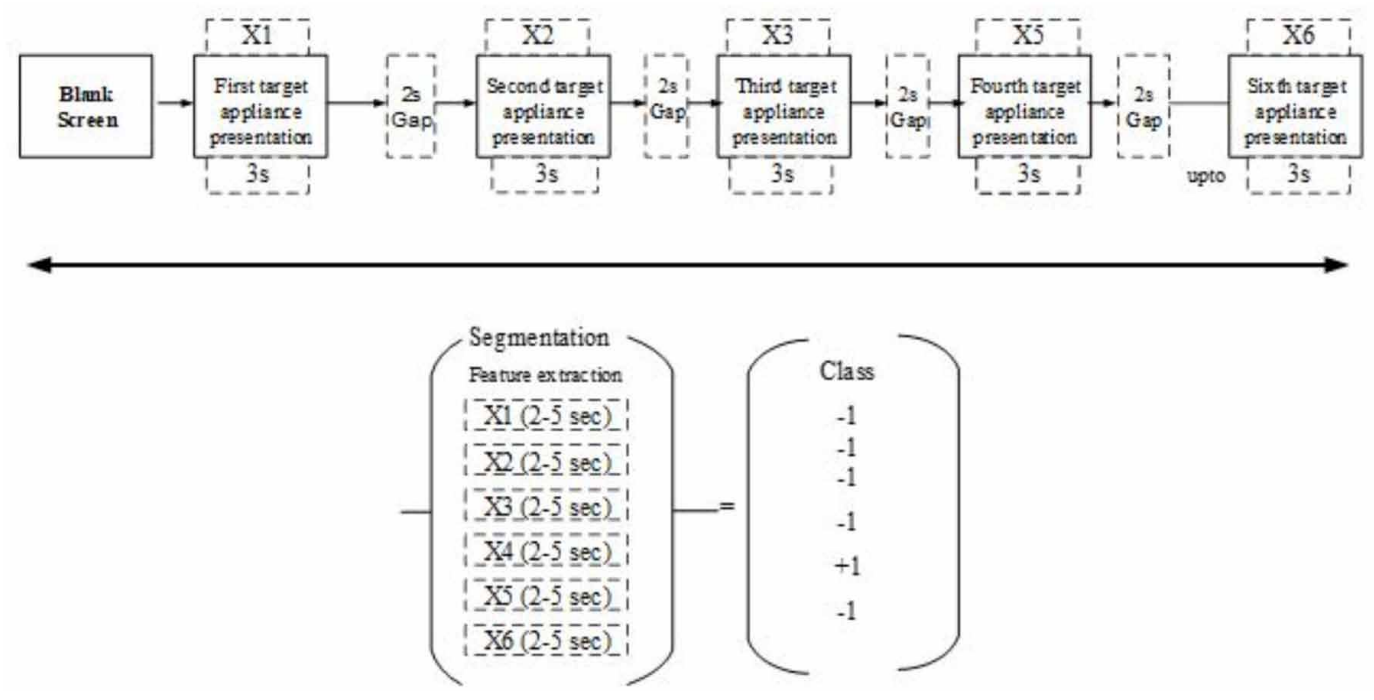

\section{Training of Acquired Data}

\section{Training of ANN Classifier}

The multilayer feed-forwards with three hidden layers with a sigmoid activation function was used in this proposed classification task. This presented study used three hidden layers (256, 5 and 2 neurons based on mean square error) and was chosen as the basis for the trial and error.

\section{Training of SVM Classifier}

In the proposed classification problem SVM classifier is trained with full training data set using the RBF-SVM classifier. The technique of 5-fold cross-validation was used. To summarize, in the RBF- 
SVM, $C$ and $\sigma$ parameters need to configure for better classification performance. The parameter of $\mathrm{C}$ and $\sigma$ (for this experiment $\mathrm{C}=26, \sigma=28$ ) were applied to get the best results.

\section{Training of LDA Classifier}

In the proposed classification task the hyperplane separation is obtained by maximizing two classes distance of their means and minimizes the interclass variance. Fisher's LDA assumes that data having a normal distribution with equal covariance matrix between the classes.

\section{Target Appliance Prediction and Hardware Design}

After segmenting and classifying the blink and non-blink signals of the training session, we have applied classifiers to classify targeted home appliances. Ideally, as a result of classification, we should have two features vectors of blink (class +1 ) and non-blink (class -1 ). But the data is too noisy to get the correct target appliance from just one trial. So we used a multi-trial approach to reduce this issue. We added +1 to the score of detected in class +1 after each trial. Therefore, after $n$ number of trails, the highest score was listed to represent the desired appliance.

After the detection of a target appliance, signals are transmitted through a Bluetooth module (HC-05) to the Arduino module. Specifications of the HC-05 module are, voltage range $=4 \mathrm{~V}$ to $6 \mathrm{~V}$ (Usually $+5 \mathrm{~V}$ ) and current can flow up to $30 \mathrm{~mA}$. The Bluetooth module is working with serial communication and follows the IEEE 802.15.1 standard protocol. The collected signal is transmitted to the Arduino board using a Bluetooth module HC-05. The module supports a baud rate of 57,600 that can be selected using the AT (attention) commands. Signals received by an HC-05 Bluetooth module that is directly attached to the Arduino module. Arduino UNO board is working as a microcontroller board based on the AT mega 238. Figure 8 shows a screenshot of hardware design in real-time. It supports $16 \mathrm{MHz}$ crystal oscillator. It has 16 digital inputs and output pins, six pins are used for the PWM outputs, 6 pins are analog pins one pin is used for USB connection, a power jack, and one pin for ICSP heater. Figure 9 presents pin configuration of Bluetooth-based Arduino module. Once a blink signal detected, it is transmitted to a relay board that switched the suitable device accordingly.

\section{Proposed Algorithm}

Figure 10 shows the flow chart of the proposed work. In the proposed design, the neurosky mindwave mobile headset was used for data acquisition. Speller was shown in front of the subjects for data collection. In the speller, flashes are changed in random order. The subject can select any of the appliances according to his/her needs using eye blink. Once the eye blink and target appliance detection are complete, it will give translation command to the Arduino board which is connected through the HC-05 module. Once Arduino gets the command related to the particular target appliance, it will pass command through a relay and activate the targeted appliance.

\section{RESULTS}

The resulting segment is divided into three parts. The first one presents the results of the blink and non-blink classification using three ANN, SVM, and LDA classifier. The second presents target appliance detection accuracy results with 5,10 and 15 sequences of the signals. The third part shows the results obtained with the statistical analysis of the data. The performance of classification has been assessed in terms of accuracy. Figure 11 displays the flow chart for the classification process.

\section{Result of the Signals for Blink Classification}

For eye blink detection, LDA, SVM, and ANN models were trained and evaluated using 36 target appliances dataset. The results of the blink classification for 36 target appliance detection subjectwise and trail wise shown in Table 2. 
Figure 8. Screenshot of hardware design in real environment

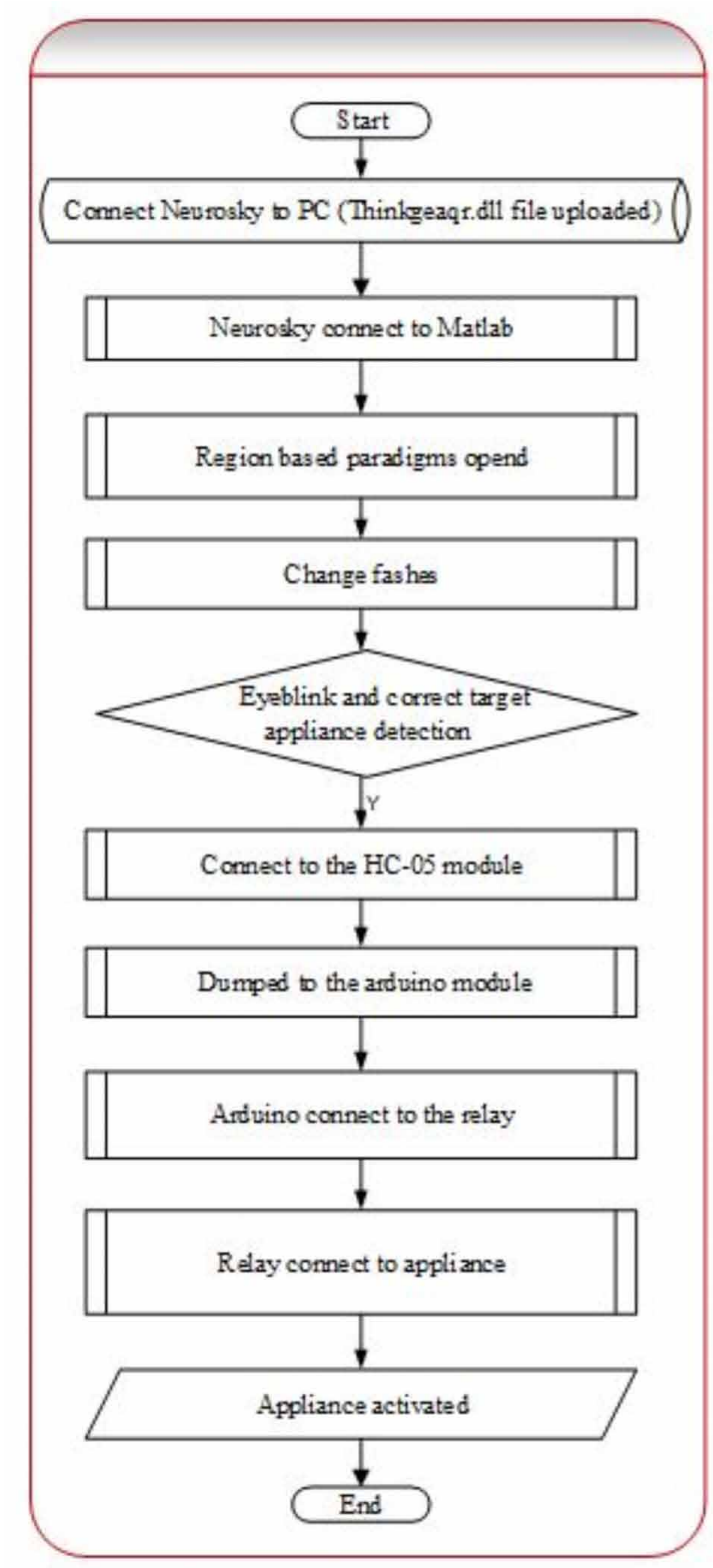

The average accuracy of subject wise and trails wise are shown in Table 2. In the case of blink detection using SVM classifier, the achieved average accuracy in 5, 10, and 15 trails are 59.00\%, $67.77 \%, 75.77 \%$ respectively.

In the case of SVM classifier, subject wise highest accuracy is achieved for subject 1 in 15 trails and the lowest accuracy was achieved by subject 9 in 5 trails. Subject S8 and S2 attained the largest average accuracy and the smallest average accuracy of $65.33 \%$, and $71 \%$ respectively. The reduced observed accuracy from 15 trials to 5 trials in percent are S1-24, S2-18, S3-17, S4-23, S5-15, S6-13, S7-11, S8-13, S9-17\% respectively. 
Figure 9. Pin configuration of Bluetooth- based Arduino control module

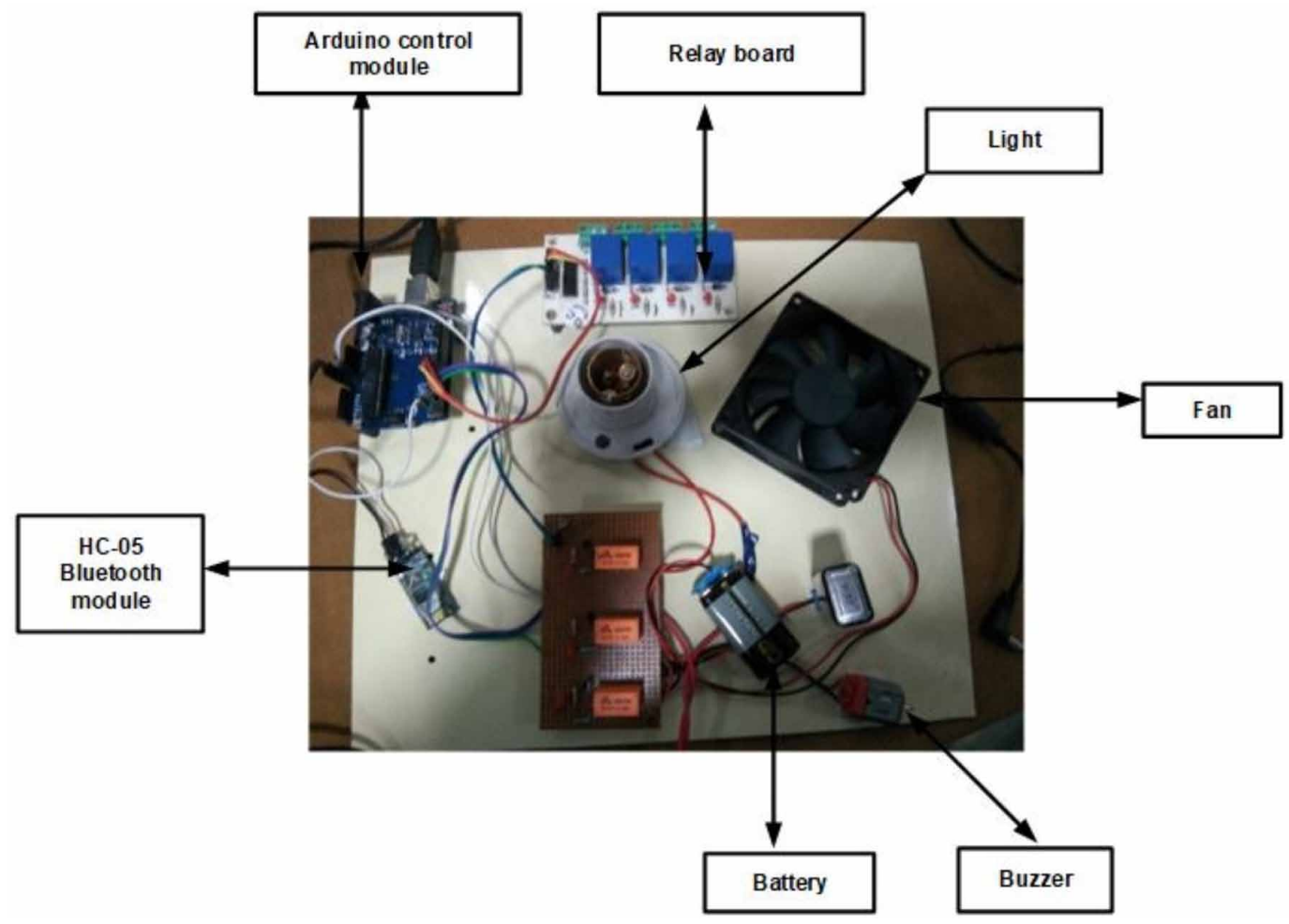

Figure 10. Flow chart of the proposed algorithms

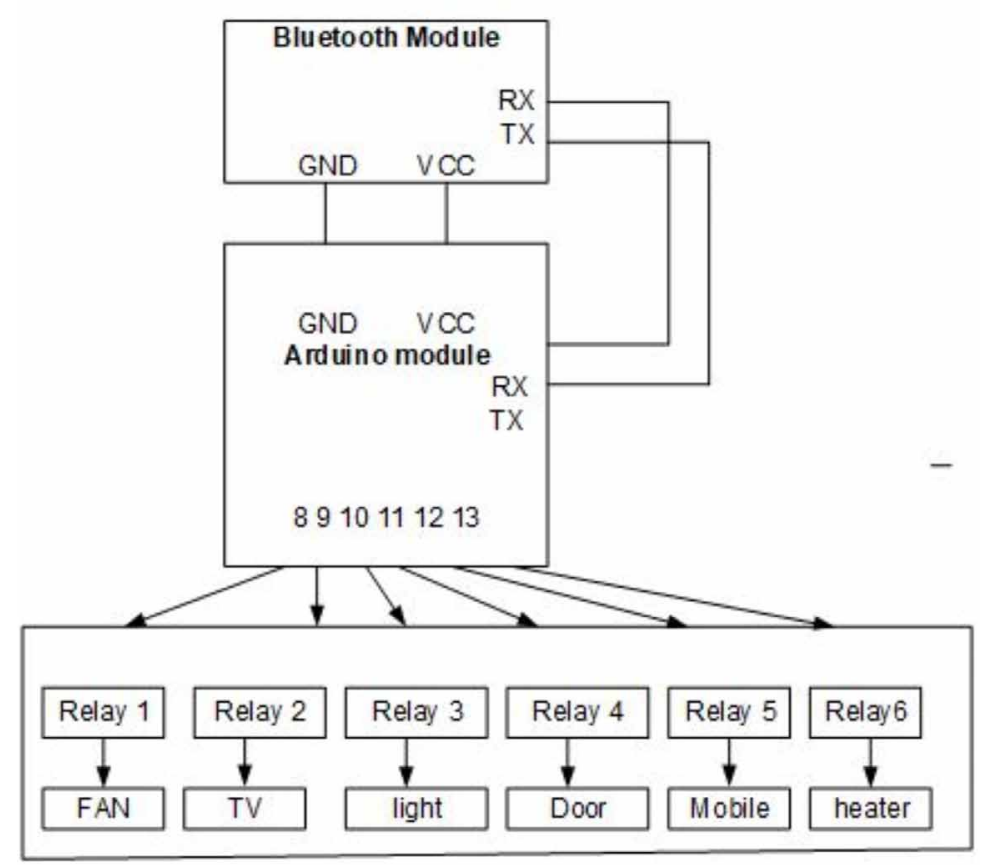


Table 2. Result obtained using the single channel with 5, 10 and 15 sequences of the signals for blink classification

\begin{tabular}{|c|c|c|c|c|c|c|c|c|c|c|}
\hline \multirow{3}{*}{$\begin{array}{c}\text { SUB. } \\
\text { NO. }\end{array}$} & \multirow{3}{*}{$\begin{array}{c}\text { User } \\
\text { Number }\end{array}$} & \multicolumn{9}{|c|}{ Classification accuracy for different subject } \\
\hline & & \multicolumn{3}{|c|}{5 Trails } & \multicolumn{3}{|c|}{10 trails } & \multicolumn{3}{|c|}{15 Trails } \\
\hline & & ANN & LDA & SVM & ANN & LDA & SVM & ANN & LDA & SVM \\
\hline 1 & Subject 1 & 59 & 56 & 53 & 70 & 65 & 68 & 80 & 71 & 77 \\
\hline 2 & Subject 2 & 58 & 52 & 56 & 70 & 62 & 66 & 76 & 71 & 74 \\
\hline 3 & Subject 3 & 61 & 51 & 59 & 71 & 66 & 67 & 77 & 69 & 76 \\
\hline 4 & Subject 4 & 63 & 57 & 53 & 71 & 65 & 69 & 76 & 70 & 76 \\
\hline 5 & Subject 5 & 64 & 62 & 58 & 72 & 67 & 66 & 78 & 68 & 73 \\
\hline 6 & Subject 6 & 65 & 63 & 62 & 73 & 69 & 69 & 82 & 74 & 75 \\
\hline 7 & Subject 7 & 60 & 65 & 63 & 71 & 68 & 66 & 84 & 72 & 74 \\
\hline 8 & Subject 8 & 68 & 56 & 65 & 70 & 66 & 70 & 83 & 71 & 78 \\
\hline 9 & Subject 9 & 67 & 59 & 62 & 73 & 65 & 69 & 79 & 72 & 79 \\
\hline & Average & 62.77 & 57.88 & 59.00 & 71.22 & 65.88 & 67.77 & 79.44 & 70.88 & 75.77 \\
\hline
\end{tabular}

In the case of blink detection using the LDA classifier, the average accuracy in 5, 10 and 15 trails are $57.88 \%, 65.88 \%$, and $70.88 \%$ respectively. Subject 6 was achieved in 15 trails and subject 3 was achieved the lowest accuracy in 5 trails. Subject 2 and attained the largest average accuracy of $69 \%$ and subject 6 attained the smallest $62 \%$ respectively. The reduced observed accuracy from 15 trials to 5 trials in percent are S1-15\%, S2-19\%, S3-18\%, S4-13\%, S5-6\%, S6-11\%, S7-7\%, S8-15\%, S9-13\% respectively.

In the case of blink detection using ANN classifier, the average accuracy in 5, 10 and 15 trails are $62 \%, 71.22 \%$, and $79.44 \%$ respectively. Subject 5 was achieved in 15 trails and the lowest accuracy was achieved by subject 2 in 5 trails. Subject 2 attained the smallest average accuracy of $68 \%$ and subject 6 attained the highest accuracy $74 \%$ respectively. The reduced observed accuracy from 15 trials to 5 trials in percent is $\mathrm{S} 1-18 \%, \mathrm{~S} 2-16 \%, \mathrm{~S} 3-15 \%, \mathrm{~S} 4-13 \%, \mathrm{~S} 5-9 \%, \mathrm{~S} 6-10 \%, \mathrm{~S} 7-14 \%, \mathrm{~S} 8-10 \%$, $\mathrm{S} 9-12 \%$ respectively. The result shows that for blink classification ANN classifier gives a better result than others.

\section{Target Appliances Detection (In Region-Based)}

Target appliance detection to the corresponding blink classification is detected for a different number of trails. The results of the 36 target appliance detection classifier wise shown in Figure 12, 13, and14. Blue line shows 5 trails, the red line shows 10 trails and the green line shows 15 trails.

In the case of the target appliances detection to the corresponding blink classification using SVM, an average of all subject 19.5 24, 23.5 target appliances were detected correctly out of 36 in 5, 10 and 15 trails. However, Subject 2 has the highest appliance detection among all subjects. Subject 9 performs the lowest correctly appliance detection. However, when we lowered the number of trails from 15 to 5, the amount of target appliance detection incorrectly categorized increases. The average accuracy for target appliance detection in 5, 10 and 15 trails 54.1\%, 66.6\%, and $65.41 \%$ respectively.

In the case of the target appliances detection to the corresponding blink classification using LDA, an average of 17.5, 20,21.1. Target appliances were detected correctly out of 36 in 5, 10 and 15 trails. However, Subject 1 has the highest appliance detection among all subjects. Subject 6 performs the lowest correctly appliance detection. However, when we lowered the number of trails from 15 to 5 , the amount of target appliance detection incorrectly categorized increases. 


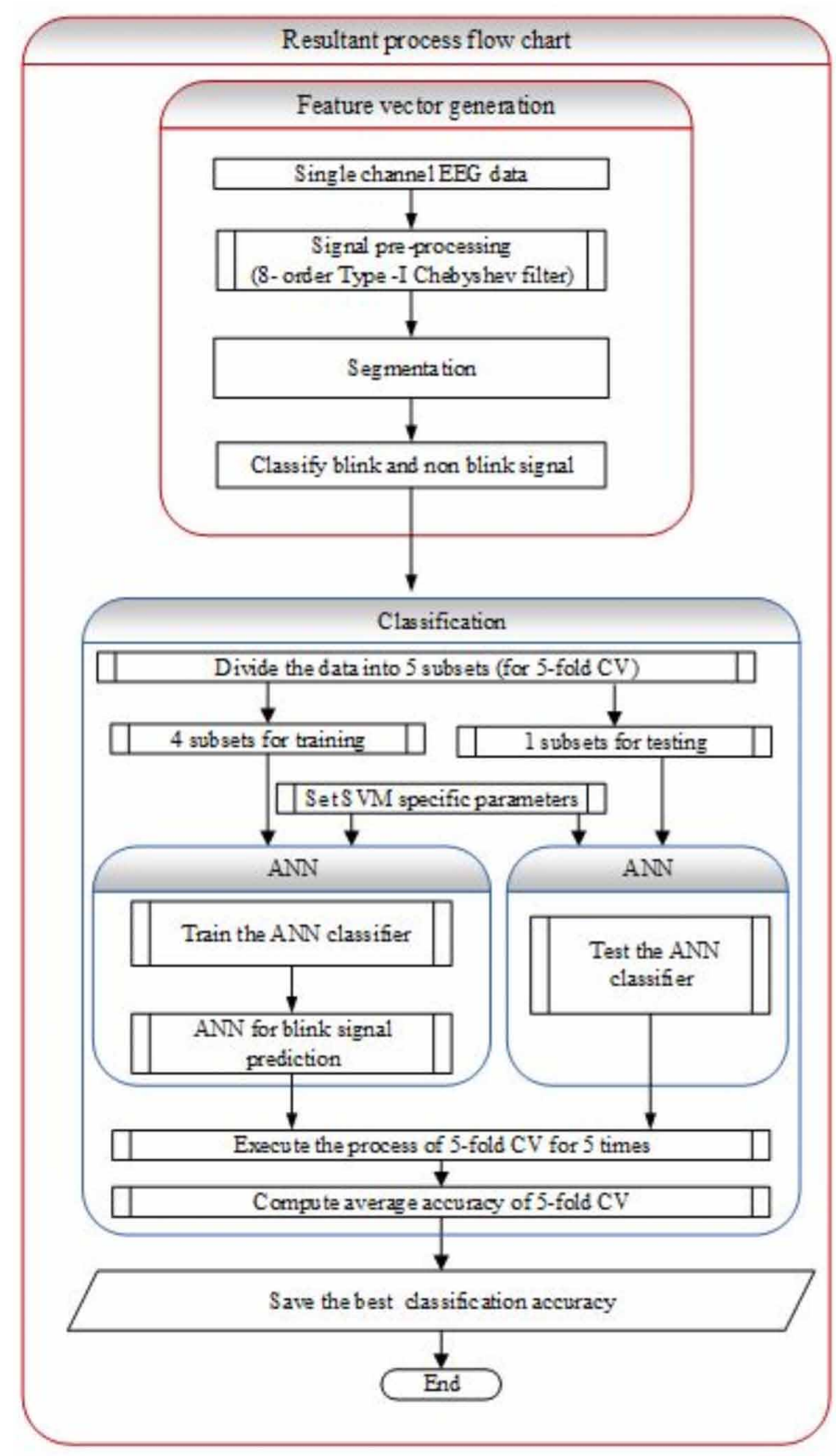

In the case of the target appliances detection to the detected blink classification using ANN, an average of 24.5, 26.55, 27.33 target appliances were detected correctly out of 36 in 5, 10 and 15 trails. However subject 4 has the highest appliance detection among all subjects. Subject 9 performs the lowest correctly appliance detection. However, when we lowered the number of trails from 15 to 5 , the amount of target appliance detection incorrectly categorized increases. The average accuracy for target appliance detection in 5, 10 and 15 trails 68.00, 73.77, and 75.9 respectively.

Figures 12, 13 and 14 show the achieved results using different classifiers on 9 subjects for 5 trails 10 trails and 15 trails. Table 3 shows the average accuracy of nine subjects in 15 trails using all used classifiers. Among all classifiers, ANN classifiers performed better than others. 
Figure 12. Target appliance detection using LDA

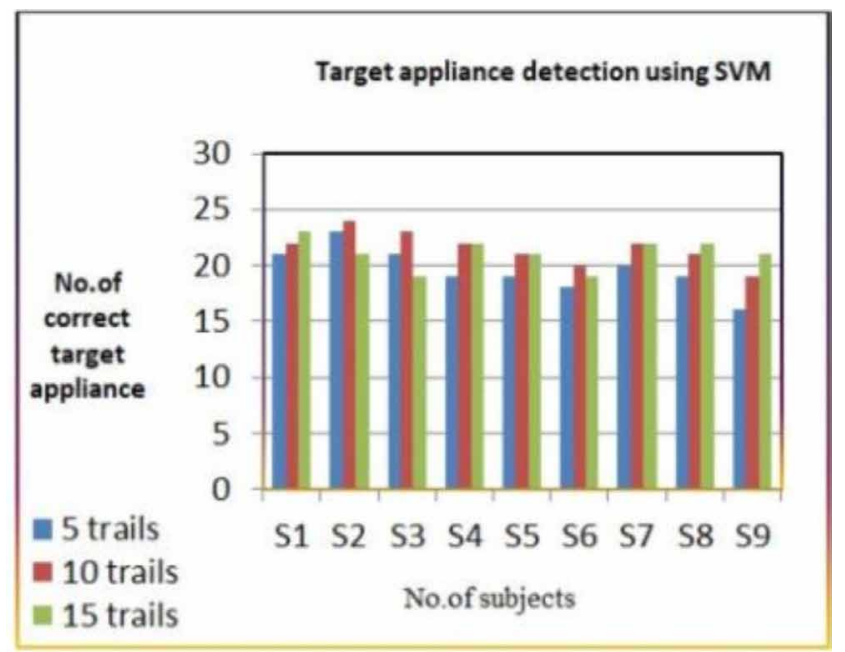

Figure 13. Target appliance detection using SVM

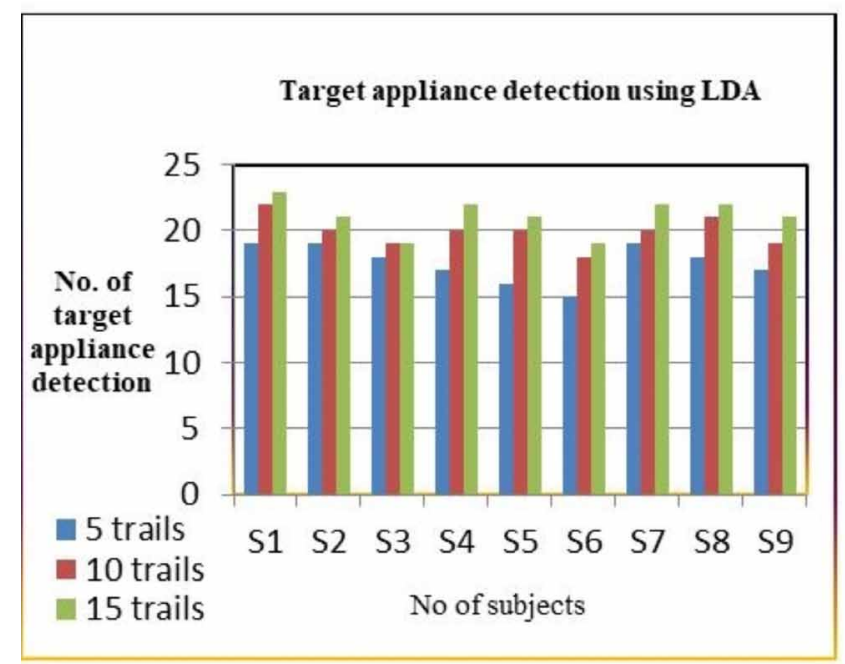

\section{Statistical Analysis}

The Friedman method was used for statistical comparison of various algorithms. It is the nonparametric method chosen over repetitive ANOVA analysis. This test can be said to be similar to repeated ANOVA analysis. The basic principle of this experiment is that various methods of classification are evaluated according to their ranking. Rank 1 rate the best performing system the second one rank 2 and so forth (Figure 15).

The Friedman method is used according to the average ranks obtained from the different approaches. A null hypothesis for the $\mathrm{p}$-value is evaluated. A p-value refers to a confidence level. The assumption is that if the p-value is higher than the confidence level then there is no distinction between subjects, the answer will be rejected. The post hoc Nemenyi test is used in this situation. The next criterion is that if the p-value approaches the confidence level, then there is a rank difference between 
Figure 14. Target appliance detection using ANN

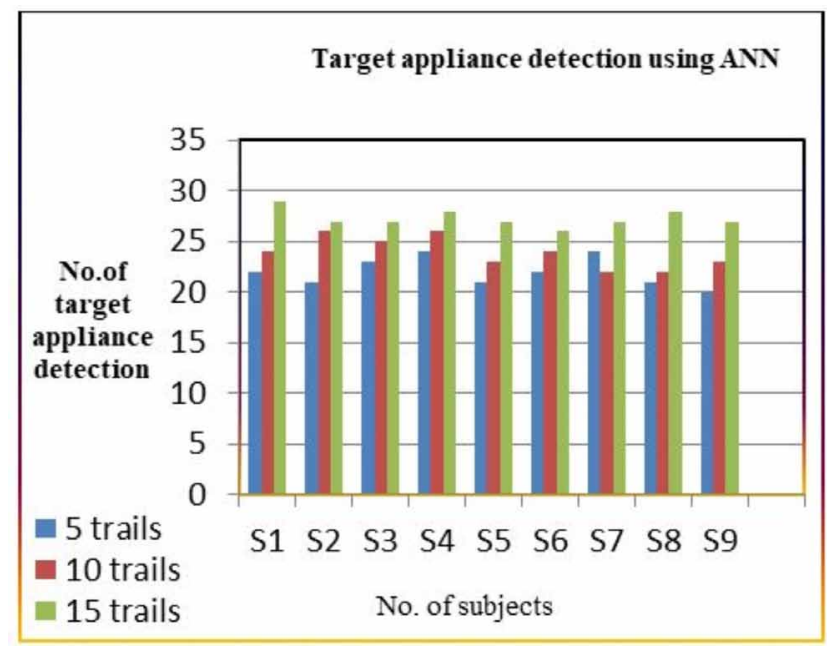

Table 3. Average accuracy of all nine subjects in 15 trails

\begin{tabular}{|l|l|l|}
\hline \multicolumn{1}{|c|}{ S.NO } & \multicolumn{1}{|c|}{ Classifier } & \multicolumn{1}{c|}{ Average accuracy } \\
\hline 1 & ANN & $80.00 \%$ \\
\hline 2 & SVM & $75.77 \%$ \\
\hline 3 & LDA & $70.88 \%$ \\
\hline 4 & KNN & $68.77 \%$ \\
\hline 5 & Bayes & $60.55 \%$ \\
\hline
\end{tabular}

Figure 15. Shows the results of the post hoc analysis for all three models

\section{Friedman $p=0.01564$}

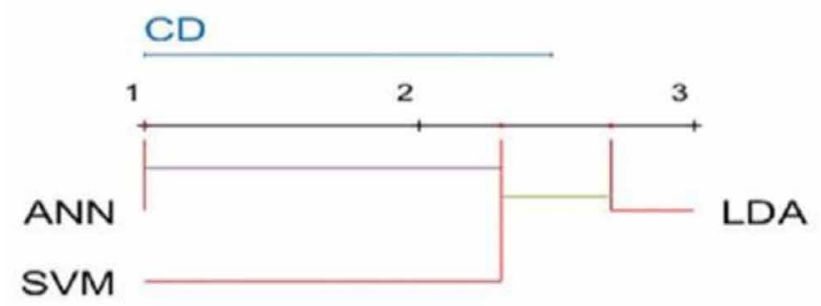

a pair being greater than the critical difference (CD) at a certain confidence level, the performance will be significantly different in this case. The Friedman method was implemented across all nine different subjects with three different classification methods (ANN, SVM, and LDA) (obtained using 15 trails). The estimated P-value according to the experimental outcome was 0.01564 lower than the 
confidence level. The null hypothesis was rejected by rule and the Post Hoc test was used. The CD value obtained is 2.5135 more than the p-value of 0.05 .

\section{DISCUSSION AND FUTURE SCOPE}

Table 4 demonstrates the comparison between the proposed and existing BCI-based controlling home appliances. We compared the accuracy as well as hardware design (cost of the system, convenience of the users) of the existing model

\section{Comparison Study Between Proposed Vs Existing Controlling Home Appliances System}

Regarding existing studies related to BCI-based home appliances control system applications, Hoffman et al. (Hoffmann, et al., 2008) tested a P300-based BCI system for 5 disabled subjects having distinct pathologies and 4 healthy subjects simulating a paradigm for environmental control. Four of them achieved $100 \%$ classification accuracy. Nevertheless, a quite distinct P300 paradigm was used to carry out this research. One stimuli matrix was used, consisting of six pictures flashing one by one. In addition, four sessions were held, each consisting of six single item selections. In the proposed BCI design system contains 36 items from 6 menus or matrices, stimuli were distributed over the intended area, and 2 assessment sessions are performed consisting of at least 36 single item selections. Rebeca Corralejo et. al(Corralejo, et al., 2014) increases 113 items from ten menus or matrices, 2 assessment sessions are conducted consisting of at least 42 choices of single items. But overall average accuracy $74.4 \%$ achieved. In our proposed BCI system based on the ANN model

Table 4. Comparative analysis between existing and proposed $\mathrm{BCl}$ - based home appliance control system

\begin{tabular}{|c|c|c|c|c|c|c|c|c|}
\hline Author & Subjects & $\begin{array}{c}\text { Types of } \\
\text { Paradigms }\end{array}$ & Classifier & $\begin{array}{c}\text { Total } \\
\text { selection }\end{array}$ & $\begin{array}{c}\text { Average } \\
\text { Accuracy (\%) }\end{array}$ & Session & Signal & Disability \\
\hline $\begin{array}{l}\text { Hoffman et al. } \\
\text { (Hoffmann, et } \\
\text { al., 2008) }\end{array}$ & 4 & $3 \times 2$ images & $\begin{array}{l}\text { Set wise-LDA, } \\
\text { Fisher-LDA }\end{array}$ & $24(2)$ & 100.0 & 2 & P300 & Healthy \\
\hline $\begin{array}{l}\text { Rebecca } \\
\text { Corralejo } \\
\text { (Corralejo, et al., } \\
\text { 2014) }\end{array}$ & 15 & $\begin{array}{l}10 \text { matrices } \\
\text { images } \\
\mathrm{R} / \mathrm{C}\end{array}$ & SLDA & $134(3)$ & 74.4 & 3 & P300 & Disabled \\
\hline $\begin{array}{l}\text { E.A.Aydin } \\
\text { (Aydin, et al., } \\
\text { 2016) }\end{array}$ & 5 & $\begin{array}{l}\text { Main } \\
\text { menu 6x6, } \\
\text { Submenu } \\
6 x 6\end{array}$ & LDA & $20(2)$ & $95 \%$ & 2 & P300 & Healthy \\
\hline $\begin{array}{l}\text { S.F.Anindya } \\
\text { et. al }\end{array}$ & 4 & - & SVM & - & $83.26 \%$ & - & SSVEP & Healthy \\
\hline 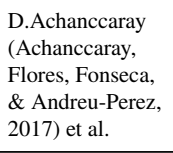 & 8 & Matrix $2 \times 3$ & ANFIS & - & Above $80 \%$ & - & $\begin{array}{l}\text { Eye } \\
\text { blink } \\
\text { (EEG/ } \\
\text { EOG) }\end{array}$ & Healthy \\
\hline $\begin{array}{l}\text { M.Uma(Uma \& } \\
\text { Sheela, 2017). }\end{array}$ & 5 & $\begin{array}{l}\text { GUI based } \\
\text { menus }\end{array}$ & ANN & - & $90 \%$ & - & P300 & Healthy \\
\hline $\begin{array}{l}\text { U.Masud } \\
\text { (Masud, et al., } \\
\text { 2017) }\end{array}$ & 3 & Matrix 4x6 & Random Forest & - & $87 \%$ & - & - & Healthy \\
\hline Proposed & 9 & $\begin{array}{l}\text { Main menu } \\
2 \times 3\end{array}$ & ANN & $36(2)$ & $80 \%$ & 2 & $\begin{array}{l}\text { Eye } \\
\text { blink } \\
\text { (EEG/ } \\
\text { EOG) }\end{array}$ & Disabled \\
\hline
\end{tabular}


achieved better accuracy than existing. E. A. Aydin et al. developed a home automation control system based on a web server that achieved good accuracy by $95 \%$. The existing home automation control system based on the webserver requires constant internet access for activation. The proposed systems need not internet access consistently. (Belwafi, et al., 2017) designs a system using FPGA model, which is a critical design for the users. Our proposed design system easy and economical for the users. (Wahy \& Mansor, 2010b) also developed a 3-eye blink signal system (four seconds long) using a microcontroller (PIC16F877A), but the proposed system was designed for single appliance only. Our proposed model convenient with an electrical device and control multiple appliance control systems. (Uma \& Sheela, 2017) designed a home appliance control system model using eye blink for a healthy person. This design did not talk deeply for signal analysis and target appliance recognition and achieved $90 \%$ accuracy for a healthy person. The proposed model deeply analyzes blink classification; waveform detection and target appliance recognition for completely disable patients and achieved $80 \%$ accuracy for completely paralyzed patients. The proposed system achieves the highest eye blink classification accuracy with subject 5, which has the locomotive disease. Furthermore, highest target character classification accuracy is achieved with subject 4, which has SCI. This model not only performing good in terms of accuracy but also has robust hardware design. The system is very economical, easy to use, and makes life easy for paralyzed people.

The different device takes some time for activation. For evaluation, average activation time for all devices was calculated and all devices were analyzed with respect to this average time. Light and fan take minimum average time for activation. The TV appliance takes maximum average time for activation. Heater and door take above average time for activation. Figure 16 shows performance of difference appliance (In terms of time).

Figure 16. Performance of different device (In terms of time)

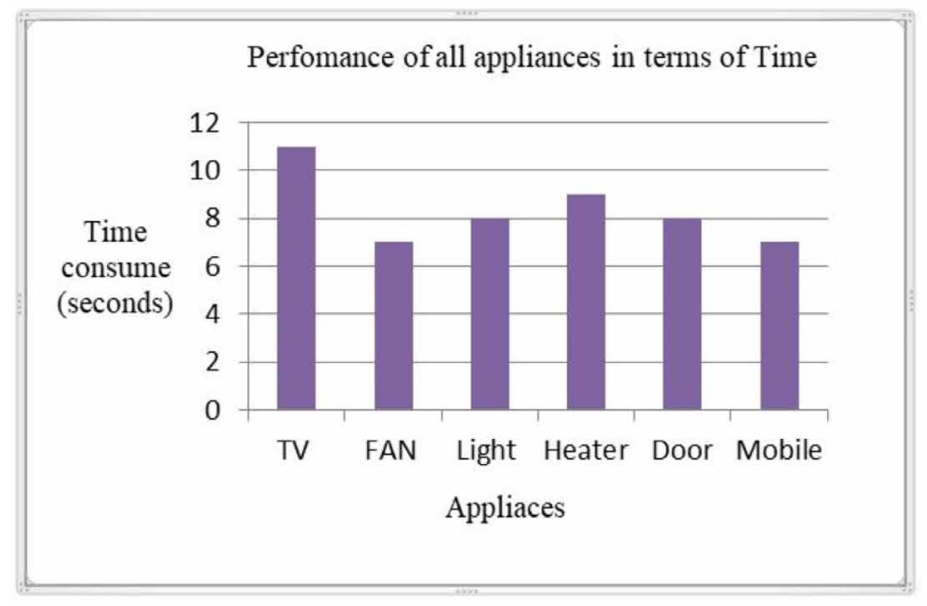

From the presented results and discussion, it is evident that further research and technical enhancement is required on EEG-based control systems for them to be publically accepted. We assume that seeing the potential of this area, the managers, government bodies and policymakers will start to focus on this in the very near future.

\section{Open Issues and Future Scope}

There are many issues and challenges in the development of BCI-based technology. These challenges are categorized based on usability and technical requirements. Non-linear behavior of the brain, 
correct data acquisition, noises and high dimensionality of the data comes under technical challenges. Information transfer rate (ITR) and the average time for selection comes under the usability challenges. RC-based paradigms are having many challenges like adjacency problem, crowding effect (Chaurasiya, Londhe, \& Ghosh, 2016), and task difficulty. Proposed work can significantly resolve such challenges. The integration of more devices will further improve the usability and performance of the proposed system. The inclusion of different kinds of disability into existing and collected datasets will make the proposed dataset more robust for the training of the proposed system. Additionally, the application of the Internet of things (IoT) with the proposed method will enhance the usability of the proposed system.

\section{CONCLUSION}

In this proposed article novel region-based paradigms for BCI-based home appliances control system. This design is very helpful, for people who are unable to move or unable to communicate verbally because of complete paralysis. In proposed designed an RB-based speller that contains 36 options which are generally used in daily life. These options can be selected with the help of just an eye blink. Moreover, EEG signals data were collected from paralyzed patients using a neurosky mind wave mobile headset for feature extraction to segment blink and non-blink signals. Different filters were applied to segment and classify blink and non-blink signals. After that, for target selection classification different classification methods like SVM, LDA, ANN, KNN and Bayes were applied. The highest achieved average accuracy through ANN classifier is $80 \%$ which is higher than (Corralejo, et al., 2014). For the blink classification, with ANN classifier, proposed system achieves subject wise highest accuracy from subject 5 in 15 trails and the lowest accuracy from subject 2 in 5 trails. In the case of the target appliances detection using ANN, an average of 24.5, 26.55, 27.33 target appliances were detected correctly out of 36 in 5, 10 and 15 trails. It is observed that the newly developed system is cost-effective and necessitates only little effort to select the target. A statistical analysis reveals that the ANN classifier is outperforming SVM, LDA, KNN, and Bayes classification methods. The Arduino-based hardware module of the proposed system is easily available and very cheap. 


\section{REFERENCES}

Achanccaray, D., Flores, C., Fonseca, C., \& Andreu-Perez, J. (2017). A p300-based brain computer interface for smart home interaction through an anfis ensemble. Paper presented at the 2017 IEEE International Conference on Fuzzy Systems (FUZZ-IEEE).

Acqualagna, L., \& Blankertz, B. (2013). Gaze-independent BCI-spelling using rapid serial visual presentation (RSVP). Clinical Neurophysiology, 124(5), 901-908. doi:10.1016/j.clinph.2012.12.050 PMID:23466266

Alrajhi, W., Alaloola, D., \& Albarqawi, A. (2017). Smart home: toward daily use of BCI-based systems. Paper presented at the Informatics, Health \& Technology (ICIHT), International Conference on. doi:10.1109/ ICIHT.2017.7899002

Alshbatat, A. I. N., Vial, P. J., Premaratne, P., \& Tran, L. C. (2014). EEG-based brain-computer interface for automating home appliances. Academic Press.

Aydın, E. A., Bay, Ö. F., \& Güler, İ. (2016). Implementation of an embedded web server application for wireless control of brain computer interface based home environments. Journal of Medical Systems, 40(1), 27. doi:10.1007/ s10916-015-0386-0 PMID:26547847

Belwafi, K., Ghaffari, F., Djemal, R., \& Romain, O. (2017). A Hardware/Software Prototype of EEG-based BCI System for Home Device Control. Journal of Signal Processing Systems for Signal, Image, and Video Technology, 89(2), 263-279. doi:10.1007/s11265-016-1192-8

Casarotto, S., Bianchi, A. M., Cerutti, S., \& Chiarenza, G. A. (2004). Principal component analysis for reduction of ocular artefacts in event-related potentials of normal and dyslexic children. Clinical Neurophysiology, 115(3), 609-619. doi:10.1016/j.clinph.2003.10.018 PMID:15036057

Chaurasiya, R. K., Londhe, N. D., \& Ghosh, S. (2016). Binary DE-based channel selection and weighted ensemble of SVM classification for novel brain-computer interface using Devanagari script-based P300 speller paradigm. International Journal of Human-Computer Interaction, 32(11), 861-877. doi:10.1080/10447318.2016.1203047

Chowdhury, A. M., Kashem, F. B., Hossan, A., \& Hasan, M. M. (2017). Brain controlled assistive buzzer system for physically impaired people. Paper presented at the Electrical, Computer and Communication Engineering (ECCE), International Conference on. doi:10.1109/ECACE.2017.7912988

Corralejo, R., Nicolás-Alonso, L. F., Álvarez, D., \& Hornero, R. (2014). A P300-based brain-computer interface aimed at operating electronic devices at home for severely disabled people. Medical \& Biological Engineering \& Computing, 52(10), 861-872. doi:10.1007/s11517-014-1191-5 PMID:25163823

Dhanalakshmi, P., Palanivel, S., \& Ramalingam, V. (2009). Classification of audio signals using SVM and RBFNN. Expert Systems with Applications, 36(3), 6069-6075. doi:10.1016/j.eswa.2008.06.126

Donoho, D. L. (2001). Sparse components of images and optimal atomic decompositions. Constructive Approximation, 17(3), 353-382. doi:10.1007/s003650010032

Farwell, L. A., \& Donchin, E. (1988). Talking off the top of your head: Toward a mental prosthesis utilizing event-related brain potentials. Electroencephalography and Clinical Neurophysiology, 70(6), 510-523. doi:10.1016/0013-4694(88)90149-6 PMID:2461285

Fazel-Rezai, R., \& Abhari, K. (2009). A region-based P300 speller for brain-computer interface. Canadian Journal of Electrical and Computer Engineering, 34(3), 81-85. doi:10.1109/CJECE.2009.5443854

Han, J., \& Moraga, C. (1995). The influence of the sigmoid function parameters on the speed of backpropagation learning. Paper presented at the International Workshop on Artificial Neural Networks. doi:10.1007/3-540-59497-3_175

He, S., \& Li, Y. (2017). A Single-channel EOG-based Speller. IEEE Transactions on Neural Systems and Rehabilitation Engineering, 25(11), 1978-1987. doi:10.1109/TNSRE.2017.2716109 PMID:28641264

Hillyard, S. A., \& Galambos, R. (1970). Eye movement artifact in the CNV. Electroencephalography and Clinical Neurophysiology, 28(2), 173-182. doi:10.1016/0013-4694(70)90185-9 PMID:4189528

Hoffmann, U., Vesin, J.-M., Ebrahimi, T., \& Diserens, K. (2008). An efficient P300-based brain-computer interface for disabled subjects. Journal of Neuroscience Methods, 167(1), 115-125. doi:10.1016/j.jneumeth.2007.03.005 PMID:17445904 
Holzner, C., Guger, C., Edlinger, G., Gronegress, C., \& Slater, M. (2009). Virtual smart home controlled by thoughts. Paper presented at the 2009 18th IEEE International Workshops on Enabling Technologies: Infrastructures for Collaborative Enterprises. doi:10.1109/WETICE.2009.41

Hsieh, K., Sun, K., Yeh, J., \& Pan, Y. (2017a). Home care by auditory Brain Computer Interface for the blind with severe physical disabilities. Paper presented at the 2017 International Conference on Applied System Innovation (ICASI). doi:10.1109/ICASI.2017.7988473

Hsieh, K., Sun, K., Yeh, J., \& Pan, Y. (2017b). Home care by auditory Brain Computer Interface for the blind with severe physical disabilities. Paper presented at the Applied System Innovation (ICASI), 2017 International Conference on. doi:10.1109/ICASI.2017.7988473

Jais, A. A., Mansor, W., Lee, K. Y., \& Fauzi, W. (2017). Motor imagery EEG analysis for home appliance control. Paper presented at the 2017 IEEE 13th International Colloquium on Signal Processing \& its Applications (CSPA).

Khushaba, R. N., Kodagoda, S., Lal, S., \& Dissanayake, G. (2011). Driver drowsiness classification using fuzzy wavelet-packet-based feature-extraction algorithm. IEEE Transactions on Biomedical Engineering, 58(1), 121-131. doi:10.1109/TBME.2010.2077291 PMID:20858575

Lin, C.-T., Lin, B.-S., Lin, F.-C., \& Chang, C.-J. (2014). Brain computer interface-based smart living environmental auto-adjustment control system in UPnP home networking. IEEE Systems Journal, 8(2), 363-370. doi:10.1109/JSYST.2012.2192756

Ma, J., Zhang, Y., Cichocki, A., \& Matsuno, F. (2014). A novel EOG/EEG hybrid human-machine interface adopting eye movements and ERPs: Application to robot control. IEEE Transactions on Biomedical Engineering, 62(3), 876-889. doi:10.1109/TBME.2014.2369483 PMID:25398172

Ma, T., Li, H., Deng, L., Yang, H., Lv, X., Li, P., Li, F., Zhang, R., Liu, T., Yao, D., \& Xu, P. (2017). The hybrid BCI system for movement control by combining motor imagery and moving onset visual evoked potential. Journal of Neural Engineering, 14(2), 026015. doi:10.1088/1741-2552/aa5d5f PMID:28145274

Mak, J. N., \& Wolpaw, J. R. (2009). Clinical applications of brain-computer interfaces: Current state and future prospects. IEEE Reviews in Biomedical Engineering, 2, 187-199. doi:10.1109/RBME.2009.2035356 PMID:20442804

Masud, U., Baig, M. I., Akram, F., \& Kim, T.-S. (2017). A P300 brain computer interface based intelligent home control system using a random forest classifier. Paper presented at the 2017 IEEE Symposium Series on Computational Intelligence (SSCI).

Mitra, S., \& Pal, S. K. (1995). Fuzzy multi-layer perceptron, inferencing and rule generation. IEEE Transactions on Neural Networks, 6(1), 51-63. doi:10.1109/72.363450 PMID:18263285

Pan, J., Li, Y., Gu, Z., \& Yu, Z. (2013). A comparison study of two P300 speller paradigms for brain-computer interface. Cognitive Neurodynamics, 7(6), 523-529. doi:10.1007/s11571-013-9253-1 PMID:24427224

Patel, R., Janawadkar, M. P., Sengottuvel, S., Gireesan, K., \& Radhakrishnan, T. S. (2016). Suppression of eyeblink associated artifact using single channel EEG data by combining cross-correlation with empirical mode decomposition. IEEE Sensors Journal, 16(18), 6947-6954. doi:10.1109/JSEN.2016.2591580

Pesin, J. (2007). Detection and removal of eyeblink artifacts from EEG using wavelet analysis and independent component analysis. Academic Press.

Qian, N. (1999). On the momentum term in gradient descent learning algorithms. Neural Networks, 12(1), 145-151. doi:10.1016/S0893-6080(98)00116-6 PMID:12662723

Ramadan, R. A., \& Vasilakos, A. V. (2017). Brain computer interface: Control signals review. Neurocomputing, 223, 26-44. doi:10.1016/j.neucom.2016.10.024

Somers, B., Francart, T., \& Bertrand, A. (2018). A generic EEG artifact removal algorithm based on the multi-channel Wiener filter. Journal of Neural Engineering, 15(3), 036007. doi:10.1088/1741-2552/aaac92 PMID:29393057

Soomro, M. H., Badruddin, N., Yusoff, M. Z., \& Jatoi, M. A. (2013). Automatic eye-blink artifact removal method based on EMD-CCA. Paper presented at the 2013 ICME International Conference on Complex Medical Engineering. doi:10.1109/ICCME.2013.6548236 
Sweeney, K. T., Ward, T. E., \& McLoone, S. F. (2012). Artifact removal in physiological signals-Practices and possibilities. IEEE Transactions on Information Technology in Biomedicine, 16(3), 488-500. doi:10.1109/ TITB.2012.2188536 PMID:22361665

Tibdewal, M. N., Fate, R., Mahadevappa, M., \& Ray, A. (2015). Detection and classification of eye blink artifact in electroencephalogram through discrete Wavelet Transform and neural network. Paper presented at the 2015 International Conference on Pervasive Computing (ICPC). doi:10.1109/PERVASIVE.2015.7087077

Townsend, G., LaPallo, B., Boulay, C., Krusienski, D., Frye, G., Hauser, C., Schwartz, N. E., Vaughan, T. M., Wolpaw, J. R., \& Sellers, E. W. (2010). A novel P300-based brain-computer interface stimulus presentation paradigm: Moving beyond rows and columns. Clinical Neurophysiology, 121(7), 1109-1120. doi:10.1016/j. clinph.2010.01.030 PMID:20347387

Tseng, K. C., Wang, Y.-T., Lin, B.-S., \& Hsieh, P. H. (2012). Brain computer interface-based multimedia controller. Paper presented at the Intelligent Information Hiding and Multimedia Signal Processing (IIH-MSP), 2012 Eighth International Conference on. doi:10.1109/IIH-MSP.2012.73

Uma, M., \& Sheela, T. (2017). Analysis of Collaborative Brain Computer Interface (BCI) based Personalized GUI for Differently Abled. Intelligent Automation \& Soft Computing, 1-11.

Usakli, A. B., \& Gurkan, S. (2010). Design of a novel efficient human-computer interface: An electrooculagram based virtual keyboard. IEEE Transactions on Instrumentation and Measurement, 59(8), 2099-2108. doi:10.1109/TIM.2009.2030923

Velayutham, B., Kangusamy, B., Joshua, V., \& Mehendale, S. (2016). The prevalence of disability in elderly in India-Analysis of 2011 census data. Disability and Health Journal, 9(4), 584-592. doi:10.1016/j. dhjo.2016.04.003 PMID:27174073

Wahy, N., \& Mansor, W. (2010a). EEG based home lighting system. Paper presented at the 2010 International Conference on Computer Applications and Industrial Electronics. doi:10.1109/ICCAIE.2010.5735107

Wahy, N., \& Mansor, W. (2010b). EEG based home lighting system. Paper presented at the Computer Applications and Industrial Electronics (ICCAIE), 2010 International Conference on. doi:10.1109/ICCAIE.2010.5735107

Wang, M., Lv, Y., Wen, M., He, S., \& Wang, G. (2016). A Fan Control System Base on Steady-State Visual Evoked Potential. Paper presented at the Computer, Consumer and Control (IS3C), 2016 International Symposium on. doi:10.1109/IS3C.2016.31

Zhang, F., Yu, H., Jiang, J., Wang, Z., \& Qin, X. (2019). Brain-computer control interface design for virtual household appliances based on steady-state visually evoked potential recognition. Visual Informatics.

Praveen Kumar Shukla received the degree in electronics and telecommunication engineering and the M.Tech. degree in control and automation from VIT Vellore, and currently pursuing Ph.D. in engineering from National Institute of Technology, Raipur. His research area includes Machine Learning, Brain-Computer Interfacing, and Biomedical Signal Processing. He has authored several research articles in aforementioned areas.

R. K. Chaurasiya received the B. Tech. degree from the Maulana Azad National Institute of Technology, Bhopal, India, in 2009, and the M.E. degree in System Science and Automation from the Indian Institute of Science, Bangalore, in 2011. He received his Ph.D. degree in 2017 from National Institute of Technology, Raipur. He was a Senior Software Engineer with Brocade Communications Systems, Bangalore, in 2011-12. During 2013-18, he was Assistant Professor at the National Institute of Technology, Raipur. Since 2019, he is with the Malviya National Institute of Technology Jaipur as Assistant Professor. His research area includes Machine Learning, Pattern Recognition, Brain-Computer Interfacing, Optimization, Biomedical Signal Processing. He has authored several research articles in aforementioned areas.

Shrish Verma received the degree in electronics and telecommunication engineering from G.E.C Jabalpur and the $M$.Tech. degree in computer engineering from IIT Kharagpur, and the Ph.D. degree in engineering from Pt. Ravi Shankar Shukla University Raipur. He is currently the Dean academic and a Professor with the Department of Electronics and Telecommunication Engineering, National Institute of Technology, Raipur. He has authored over 150 research papers in various journals and conferences in the field of computer and communication networks, distributed processing, data mining and analysis, text analytics and software engineering. 